\section{Gradientenofen für die}

\section{Bestimmung des Einbrenn- und Trocknungsverhaltens von Lacken}

it dem Gradientenofen 432 hat die Firma Erichsen jetzt ihr Portfolio an Geräten zur Oberflächenprüfung erweitert. Der Ofen eignet sich zur Beurteilung des Einbrenn- und Trocknungsverhaltens von Lack- und Pulverbeschichtungen sowie von Harzen, Pigmenten oder Kunststoffen. Am Ofen lassen sich unter anderem Aufwärmgeschwindigkeit, Einbrenntemperatur und Zeit vorwählen, sodass sich Produktionssituationen, Einbrenn- und Trocknungsprozesse realistisch simulieren lassen. Möglich sind auch Vorhersagen darüber, wie ein bestimmtes Lacksystem bei verschiedenen Temperaturen auf ein spezielles Material reagiert. Auch die Chemikalienbeständigkeit lässt sich testen. Der Ofen bietet sich insbesondere für Rohstofflieferanten, Lackhersteller und Lackverarbeiter an. Er zeichnet sich durch gute Wiederholbarkeit der Messungen und eine genaue Bestimmung der vorliegenden Grenzwerte aus. Je nach Ofentyp können Beschichtungen bis zu einer Temperatur von $320^{\circ} \mathrm{C}$ getestet werden. Tel. 02372 9683-0, www.erichsen.de

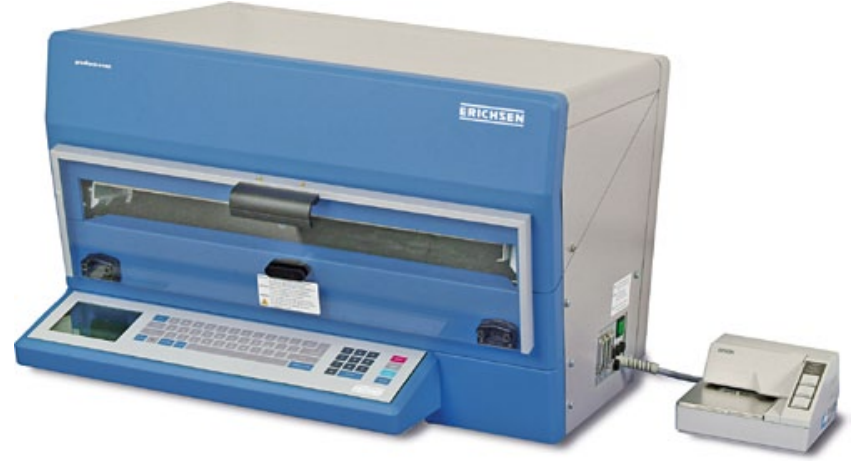

\section{Einfache Analyse von galvanischen}

\section{Prozessen}

Die e Firma Gravitech hat mit ihrem TitriQuick-AnalyticalControl TAC ein einfach zu handhabendes Analysensystem für verschiedene galvanische Prozesse entwickelt. Das Gerät eignet sich für die QS-konforme Überwachung von Prozessen wie Eloxieren, Elektropolieren oder chemisch Nickel im 24-h-Betrieb. Es besteht aus einer Waage mit Magnetrührer und einer Steuereinheit mit Software. Die Analysen werden mithilfe der gravimetrischen Titration durchgeführt. Dabei kommt das Gerät ohne Volumenmessund Dosiergeräte aus. Es führt den Anwender Schritt für Schritt durch den Analysengang. Die Ergebnisse werden gespeichert und als Konzentrations-Zeit-Diagramm angezeigt. Die Bedienung ist so einfach, dass die Analysen auch von eingewiesenem Personal ohne Laborausbildung durchgeführt werden können.

Tel. 06106 8767-71, www.gravitech.de

\section{CHAFNER (B)}

Wir suchen für unsere Galvanik eine/n

\section{Assistent/-in}

des Betriebsleiters Galvanik

Ihre Aufgabe ist die Unterstützung des Betriebsleiters Galvanik bei folgenden Aufgaben:

- Steuerung, Überwachung und Sicherung der Produktionsprozesse

- Anwendungstechnik

- Betreuung der Bestandskunden und Neukundenakquise

- Betreuung von Kundenprojekten

- Vorausschauende Personaldisposition und Auftragsterminierung

\section{Ihre Qualifikation}

- Sie sind Ingenieur für Oberflächentechnik, bzw. haben Maschinenbau mit Schwerpunkt Oberflächentechnik studiert

- Alternativ haben Sie eine Ausbildung zum Galvaniseur mit entsprechender Weiterbildung zum Meister / Galvanotechniker absolviert

- Hohe Kunden- und Qualitätsorientierung, sowie eine team- und lösungsorientierte Arbeitsweise sind für Sie selbstverständlich

ש Eigenverantwortliches und selbständiges Handeln runden Ihr Profil ab

- Gute PC-Kenntnisse, SAP/R3 Kenntnisse von Vorteil

Wir bieten

Eine interessante und anspruchsvolle Tätigkeit in einem modernen Arbeitsumfeld mit kurzen Entscheidungswegen eines Familienunternehmens.

Interessiert? Dann freuen wir uns auf Ihre Bewerbungsunterlagen. Für weitere Fragen steht Ihnen unsere Personalleiterin Frau Solveigh Dürr gerne zur Verfügung.

Seit über 160 Jahren steht der Name C.HAFNER für innovative und führende Technologien im Bereich Edelmetall. Hochmodernes Edelmetall-Recycling, digitale Dentaltechnologien, moderne Bearbeitungszentren, technische und dekorative Beschichtungen, ein breites Halbzeugespektrum sowie profundes Wissen in den unterschiedlichsten Anwendungsbereichen, bilden die Grundlage für die hohe Technologieund Lösungsorientierung von C.HAFNER.

C.HAFNER GmbH + Co. KG

Gold- und Silberscheideanstalt

Bleichstraße 13-17

75173 Pforzheim

Telefon +497231920-190

solveigh.duerr@c-hafner.de c-hafner.de 